\title{
High Performance Processing of Satellite Data Using Distributed and Parallel Computing Techniques
}

\author{
Lalit B. Damahe1, Sanket S. Bramhe ${ }^{2}$, Nilay C. Fursule ${ }^{3}$, \\ Ram D. Shirbhate ${ }^{4}$, Pournima S. Ajmire ${ }^{5}$, Girish Kumar ${ }^{6}$ \\ 1,2,3,4,5 Department of Computer Technology, Yeshwantrao Chavan College of Engineering, Nagpur, India \\ ${ }^{6}$ Scientist Engineer, RRSC-Central, ISRO, Nagpur, India
}

\begin{abstract}
In today's world of technological revolution when the volume of the data is increasing enormously coincided with the growth in technology, it has become crucial to process and store data adroitly. Due to increasing demand of high processing speed, the traditional methods of processing satellite data have become incompetent. This propelled the need for high performance computing, which is the ability to process data and complex calculations at an accelerated speed effectively and accurately. It takes prolonged time for batch processing of satellite images which acts as the foundation of analysis developments in many technological and geological fields. In this paper, presented, a proposed distributed and parallel computation solutions for satellite image processing and computation of various indices normalized difference vegetation index that improves the performance of the system. By taking advantage of apache spark and cluster computing techniques real-time high-speed stream processing of satellite data is achieved. Some main features are discussed comprehensively about apache spark cluster formation, distributive and parallel computing methodologies, calculation and processing of indices with satellite data of Landsat 5. Also, python programs for processing of satellite data of Landsat 5 are executed and their results are presented in terms of processing speed and time.
\end{abstract}

KEY WORDS: APACHE SPARK; DISTRIBUTIVE COMPUTING; HIGH-PERFORMANCE COMPUTING; PARALLEL COMPUTING; SATELLITE DATA.

\section{INTRODUCTION}

Most important method for directly obtaining information on the Earth's surface is remote sensing. Remote sensing information frequently vary features with respect to their resolution, spectrum, mode of imaging and revisited cycle (Liu, 2015) which plays a vital role in many fields of research. the possibility to gain remote detecting information has been created to a striking level. For

\section{ARTICLE INFORMATION}

*Corresponding Author: damahe l@rediffmail.com

Received 17th Oct 2020 Accepted after revision 25th Dec 2020

Print ISSN: 0974-6455 Online ISSN: 2321-4007 CODEN: BBRCBA

Thomson Reuters ISI Web of Science Clarivate Analytics USA and Crossref Indexed Journal

\section{Clarivate
Analytics}

NAAS Journal Score 2020 (4.31)

A Society of Science and Nature Publication,

Bhopal India 2020. All rights reserved.

Online Contents Available at: http//www.bbrc.in/

Doi: http://dx.doi.org/10.21786/bbrc/13.14/92 a huge ground station, the volume of universal data archive could be on Exa-byte level (Liu, 2015) which makes it imperative to process it capably. Real-time high-speed stream processing demand of satellite data can never be achieved using traditional systems. Parallel computing techniques and multicore technologies were acquired to generally speed up the computing, execution and processing efficiency, for this various parallel and distributed techniques are used.

High-performance Computing HPC) is the supercomputers application that solves computational problems that are either huge for traditional computers or time consuming. Unlike desktop PC's with single processing chip, HPC system is network of nodes with multiple processing chips and memory. HPC satisfies the demand of increasing processing speed. HPC innovation is executed in several multidisciplinary fields. HPC works on high-data transfer capacity, low-latency network to connect numerous clusters and nodes. In parallel system, numerous

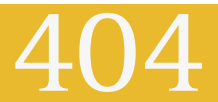


processors correspond with one another utilizing a mutual memory however in distributed system, numerous processors with individual memories are connected by network communication.

Distributed Computing: In the distributed computation as shown in figure 1, multiple computers with individual memories communicate with each other in network to perform computation tasks efficiently. Numerous processors correspond with one another using message passing techniques in the cluster (Distributed and Parallel computing, 2020) in distributed computing. Coordinating heterogeneous applications that are established and works with various operating systems and technologies, many applications having shared communal assets, a solitary occurrence administration being reused by numerous clients, and using same user interface for many functions are some of the capabilities of distributed system.Advantages of Distributed Systems are as follows:

Figure 1: Distributed Computing Architecture

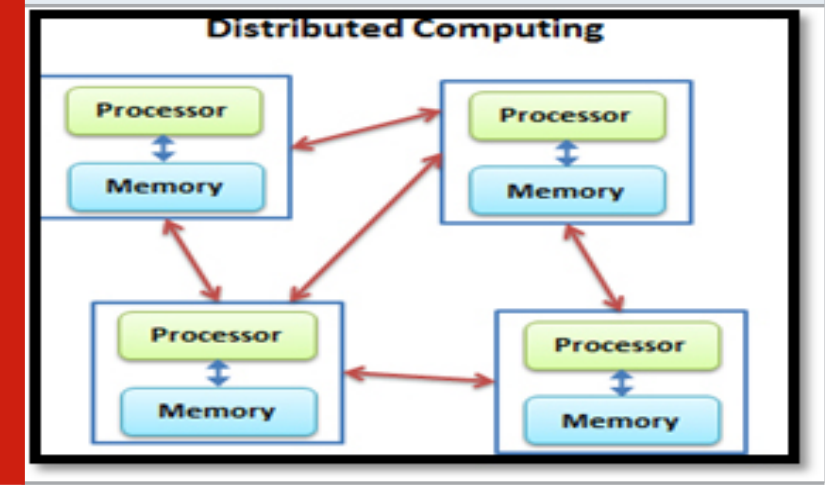

Figure 2: Parallel Computing Architecture

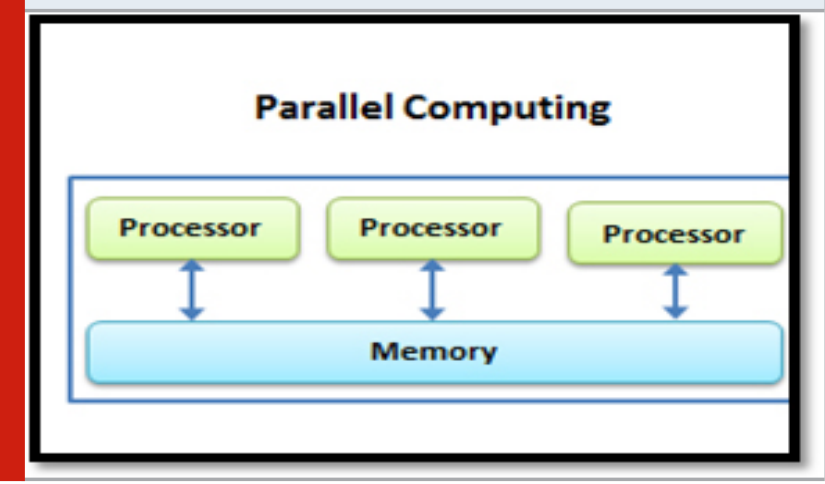

It is scalable means more nodes can be added whenever needed to increase the speed and efficiency. All nodes in distributed system are connected to every alternative in share data likewise (Distributed and Parallel computing, 2020). Thus, the failure of one node doesn't cause the failure of the distributed system as alternative nodes will still correspond with one another.

Parallel Computing: A computation type in which numerous processors carry out numerous assignments concurrently for the rapid computing of data using shared memory forming a communal address space is parallel computing. The projects requiring complex computations and more time particularly uses parallel processing technique (Distributed and Parallel computing, 2020) .In parallel processing, as shown in figure 2, numerous computations can be executed concurrently for lessening the speed of processing. The assets can incorporate a solitary PC with various processors or different PCs in system or blend of both. Advantages of Parallel Systems are as follow:

It increases the efficiency of the system as it uses all the cores of the system which reduces the time of processing.

Apache Spark: Apache spark is an open-source fast clustering framework. Spark has an in-memory computation feature, but it does not store complete data in memory. RDD (resilient distributed datasets) are created in a spark which is mainly responsible for the spark's high performance. RDD's are immutable datasets (Almeer, 2012) which are divided into various logical partitions and can be executed in the cluster.

Important Feature of Apache Spark: Speed - the logistic regression compared between Hadoop and Spark, the latter is a hundred times faster than Hadoop and ten times faster when running on disk. Apache spark provides a great speed for computing the data rapidly. Supports numerous programming languages- support various languages such as Python, Java, Scala, SQL, and R in apache spark which provides ease of usability. Runs everywhere -Spark runs on the cloud or Hadoop, or in Apache Mesos, standalone, Kubernetes, it can access various data sources.

Figure 3: Apache spark Architecture

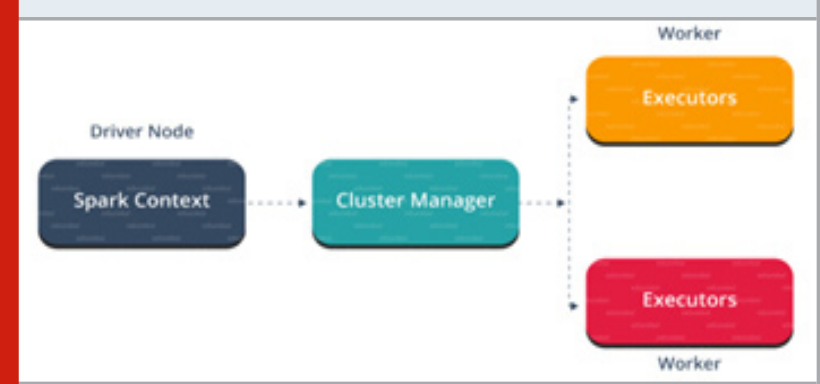

Apache Spark Architecture: As shown in figure 3, it follows the master-worker architecture in which the driver node acts as the worker node and executors act as worker nodes in the cluster. The main program of the application is called by the driver program and it creates spark-context which consists of basic functionalities. Spark-driver and spark-context collectively manage the job execution in the cluster. The cluster manager manages resource allocation. Then the job is splits into various sub-tasks that are carried out by the worker nodes (Apache Spark, 2020). When execution of tasks allocated by cluster manager is completed, it returns it back to the spark-context. 


\section{Apache Spark Components:}

Spark is improvement over the Hadoop, yet it isn't reliant on Hadoop as it has it's own cluster manager. Spark is quick and Streaming, machine learning, and graph process can be done using big data processing tools of spark as shown in figure 4.

Spark Core: It consists of the main execution engine with built in-memory computing and references to external memory dataset.

Spark SQL: It introduces the feature of schema RDD for both structured and semi-structured data (Apache Spark, 2020).

Spark Streaming: It adds the feature of real-time computation.

GraphX: It supports the manipulation and computation on graphs

Figure 4: Apache spark components

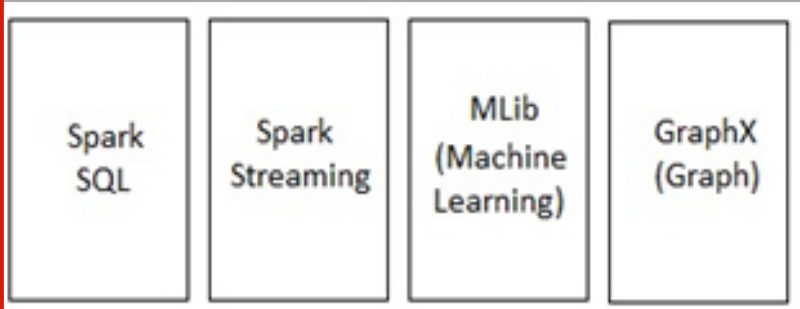

Apache Spark Core

Satellite Data: The satellite image data is obtained by the process of remote sensing. Sample is shown in figure 5.In this paper, we have used Landsat 5 dataset which is a low Earth orbit satellite launched on March 1, 1984 (Landsat 5, 2020).

Figure 3: UV-VIS Spectra of the compound, PA-B-ester
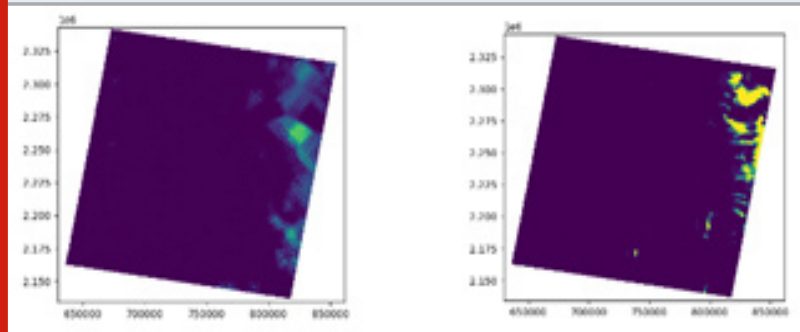

Landsat 5 dataset consists of total 10 images in Tagged image file format (TIFF) with 7 spectral band images. The 7 spectral bands consist of:

Band1- Visible Blue $(0.45-0.52 \mu \mathrm{m}) 30 \mathrm{~m}$

Band2- Visible Green $(0.52-0.60 \mu \mathrm{m}) 30 \mathrm{~m}$

Band3- Visible Red $(0.63-0.69 \mu \mathrm{m}) 30 \mathrm{~m}$

Band4- Near-Infrared $(0.76-0.90 \mu \mathrm{m}) 30 \mathrm{~m}$

Band5- SWIR $1(1.55-1.75 \mu \mathrm{m}) 30 \mathrm{~m}$

Band6- Thermal (10.40-12.50 $\mu \mathrm{m}) 120 \mathrm{~m}$

Band7- SWIR $2(2.08-2.35 \mu \mathrm{m}) 30 \mathrm{~m}$
Normalized Difference Vegetation Index (NDVI): The Band-4 and Band-5 spectral images are used for the calculation of NDVI as Equation 1, which is a index for greenness on earth surface and classifies low and high vegetation areas.

NDVI =Rnir-Rred/Rnir+Rred

Literature Survey: There is a need of developing a solution by using fast computing methods to store and process satellite data which can improve performance and reduce processing time. This section reviews the work, of many authors in order to find reasons and solutions for the problem of processing of satellite data. In present time, there are many proposed solutions and research works is available on processing of remotely captured images on hadoop. For processing big geo TIFF format images (Sharma et al., 2017) uses a hadoop mapreduce based methodology to produce NDVI raster images . Researchers showed the solution to produce NDVI image by taking landset 8 images as input. Proposed system takes the input sequence file consist of landset 8 based geo tiff images. The arrangement uses the circulated conduct of mapreduce furthermore, HDFS. The experiment determined the scale up and speedup proportions to check the performance and demonstrated that the system performs well.

(Huiyu X et al. , 2017), Identified that parallel usage of Kauffman's initialization will enhance the outcome as well as also helps in scale up. Researcher explained that Initial cluster beginning point is affecting the k-means method which Kauffman's initialization can enhance. Nonetheless, the last formula is not quicker and hence applied on hadoop mapreduce. Hence, they arranged an answer for k-means++ algorithm whereby for different values of $\mathrm{k}$, clustering is done in distributed approach for remotely captured images. A detailed review on the development in high performance computing technology (Parallel, Distributed and Cluster Computing) as well as the fundamentals of HPC for processing and analyzing of satellite data is presented (Bhojne et al., 2013).They have offered a thoughtful perspective about how various approach can be used to solve different problems. Distributed processing is mostly helpful when more data has to be processed in less cost and minimum time. Parallel processing is particularly helpful in image fusion algorithms and hyper spectral image processing, whereas cluster computing can be applied to geo-referencing, image transformation, image mosaicking based processes (Bhojne et al., 2013).

Authors (Buche et al., 2016 ; Patel et al., 2017), has discussed a detailed review on different support vector machine-based image classification algorithms out of which GA based image classification have shown promising results. Researchers showed that as the size of the satellite images and the required time for processing is also increasing, parallel computing of graphical processing unit is a good solution. To prove the solution, authors created a normal GA on CPU as well as also on GPU and the produced outcome are reviewed and 
differentiated. Authors (Patel et al., 2015) presented, how parallel computing and distributed computing methods such as MPI, CUDA, Map reduce, HIPI, Hadoop can be used efficiently to handle enormous amount of data and to fetch and query the required runtime information as per the needs of the user application. Parallel computing model of map reduce provide high efficiency in image processing of large-scale image in distributed approach.

Remotely captured images are mostly used in different department, whose size and the time to process them are increasing day by day. Researcher showed a case study of the chinese academy of science to review how they process and save the data in database. They also explained the required hardware to query and fetch the data as well as various important points such as OS, servers, different processors and data base management systems (Haiming et al., 2011). Processed satellite images in tiff by parallel processing of image processing method on hadoop map reduce is presented (Almeer, 2012). Authors claims, hadoop map reduce is specifically created for text related processing , but enormous size of data can be efficiently processed on parallel processing hadoop map reduce (Almeer , 2012) . Experiment gave the hadoop map reduce method for land cover recognition. In the Map reduce framework, a scalable modeling system is enforced (Codella et al., 2011). The importance of compression (Damahe et al., 2019) is described for variety of application including remote sensing.

\section{PROPOSED METHODOLOGY}

The proposed system aims to develop a method to escalate the efficiency of the system for rapid processing of satellite data as Satellite image processing is resource intensive and thus takes prolonged time. The proposed system consists of two major modules which are explained in detail below.

Processing of satellite data using Distributive computing technique using Apache spark: Workfolow is shown in figure 6

Prerequisite: Java JDK, Apache Hadoop, Apache Spark, Lan or Wi-Fi enabled network of systems.

Workflow: Configuration of Apache Spark- Apache Spark is installed and configured in all the distributive systems used to create the cluster of master- worker for processing of satellite data using cluster computing technique.

Formation of Cluster- Set the master node using master system ip address in the network using spark class and then number of worker nodes can be added in the network cluster as per requirement using spark-class. Process applications (programs)-

Processing the program using spark submit in the cluster with masters ip address.

Calculation of indices (NDVI) and getting rapid efficient outcome.
Processing of satellite data using Parallel computing technique: Workfolow is shown in figure 7

Prerequisite: Python libraries-Gdal, Rasterio, Multiprocessing, OpenCV, time, OS

Workflow: Calciulating input data set and the numer of cores of the system

Split Dataset into n Equal Size Chunks-

In this, Dataset and No of cores of the computer is taken as input. Dataset is Spited into $\mathrm{N}$ equal sized chunks. So, that it can be assign as one chunk per core of the processor. the application is defined with chunk generator.

Pool of Processes base on No of Cores-

To Create No of Processes, Pool feature of Multiprocessing Library of Python has been used. The Pool class creates the Python processes on each respective core of the processor.

Assign Chunk to Process:-

The application is mapping each of the chunks to an independent process. Each Process runs independent of the other process and the results are combined after the processing of various chunks is completed. Calculation of indices (NDVI) and getting rapid efficient outcome.

Figure 6: Flow Diagram of the Distributive System

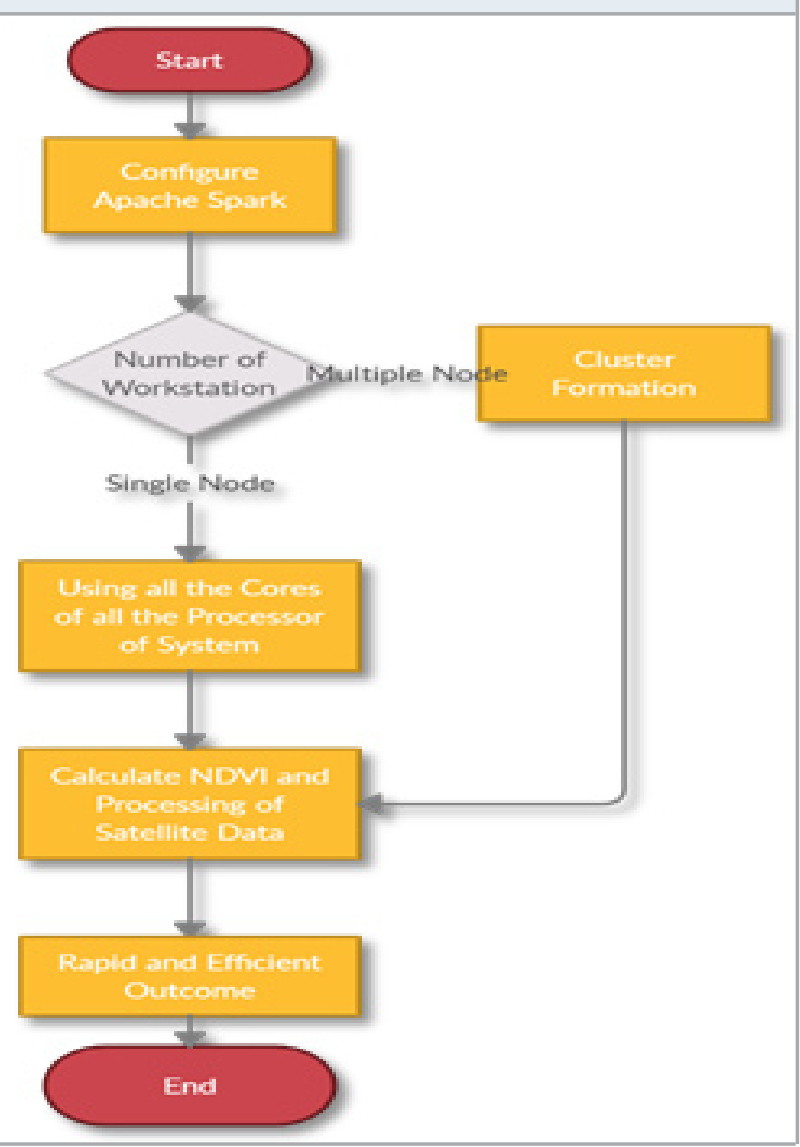


Figure 7: Flow Diagram of the Parallel System

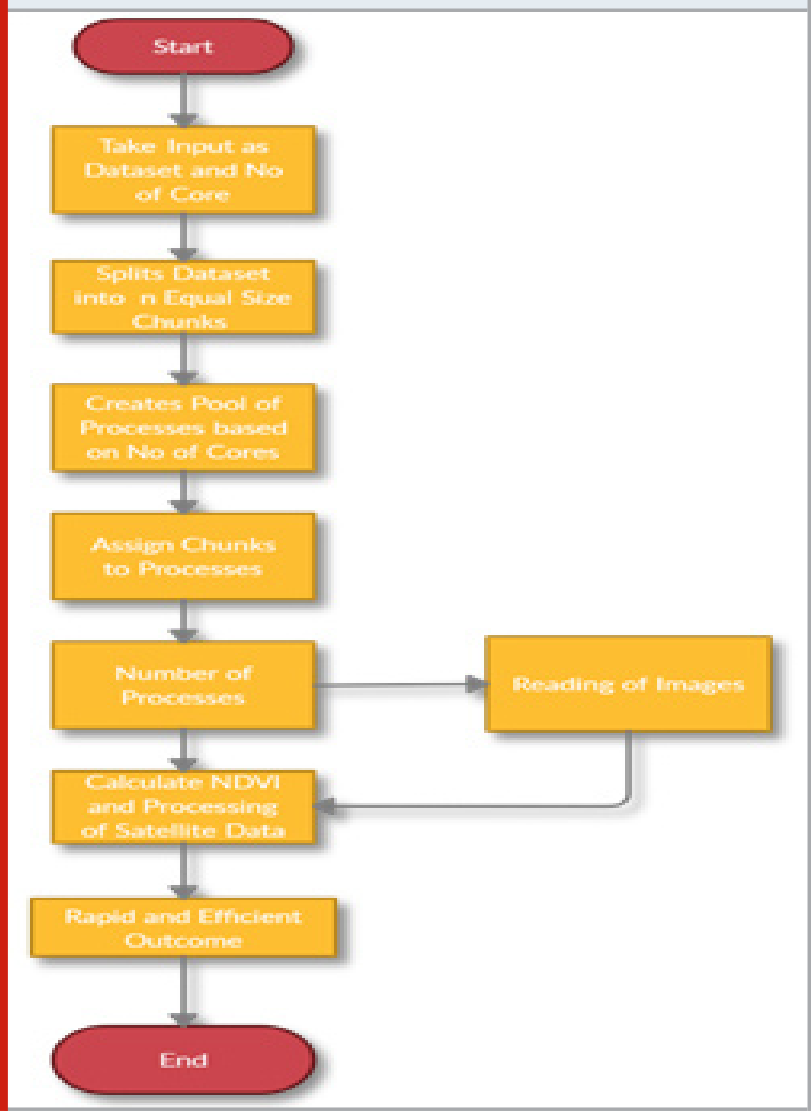

\section{RESULTS AND DISCUSSION}

Experimentation of proposed system is done on Landsat 5 dataset which contains 10 band images in tiff i.e. tagged image file format shown in figure 8. All the images are processed using parallel and distributive techniques as required and NDVI is calculated using band 3 and band 4 images using different methodologies and the processing time is reduced thus increasing efficiency. The figure below shows the pictorial view of various 10 bands images.

Figure 8: Input dataset display of Landsat 5
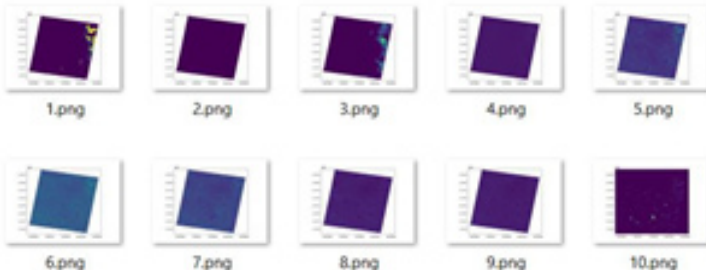

Table 1 shows the outcome of the experimentation in terms of time required for processing and calculation of NDVI on satellite data using different methodologies of distributive and parallel computing techniques along with normal computation on a single system. The outcome of the NDVI calculation is shown in figure 9, which represents the vegetation index in the required geographic area

Table 1. Comparison of DC and PC technique

\begin{tabular}{|l|c|c|c|}
\hline & $\begin{array}{c}\text { Normal } \\
\text { Computation } \\
\text { of NDVI } \\
\text { Technique }\end{array}$ & $\begin{array}{c}\text { Using } \\
\text { Distributed } \\
\text { Computing } \\
\text { Technique }\end{array}$ & $\begin{array}{c}\text { Using } \\
\text { Parallel } \\
\text { Computing }\end{array}$ \\
\hline $\begin{array}{l}\text { Time } \\
\text { Required }\end{array}$ & $\begin{array}{c}4.104 \\
\text { Seconds }\end{array}$ & $\begin{array}{c}3.00 \\
\text { Seconds }\end{array}$ & $\begin{array}{c}2.338 \\
\text { Seconds }\end{array}$ \\
\hline
\end{tabular}

Figure 9: NDVI output of programs

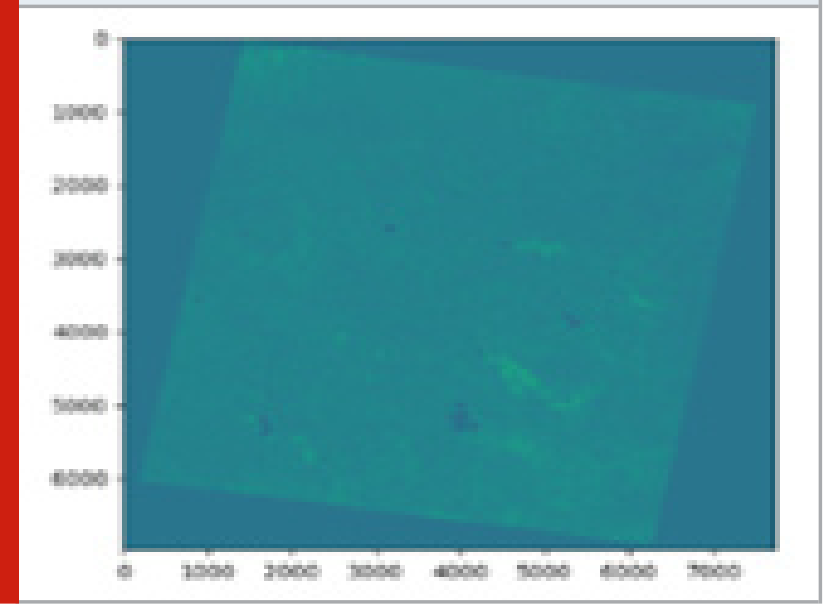

\section{CONCLUSION}

In the given approach of processing satellite data and calculation of indices using high performance computing methodologies such as parallel and distributive computing techniques that increase the computational speed and execution efficiency over existing traditional methods. The methodology to process tiff images of satellite data for calculation of various indices including NDVI at high computational speed to increase the efficiency of the system is presented. By taking advantage of apache spark and cluster computing techniques real-time highspeed stream processing of satellite data is achieved and the results are studied in terms of processing speed. In future experiments, implementation can be done on large clusters and with customized algorithms. Also processing using GPU based parallel system can be achieved.

\section{REFERENCES}

Almeer M H, "Cloud Hadoop map reduce for remote sensing image analysis”, J Emerg Trends Comput Inf Sci , Vol 3, 2012, pp 637-644.

Apache Spark, [online] Available: https://intellipaat. com/blog/tutorial/spark-tutorial/.

Bhojne M, Chakravarti A, Pallav A, Sivakumar, "High 
Performance Computing for Satellite Image Processing and Analyzing - A Review", International Journal of Computer Applications Technology and Research, 2, 2013, pp 424-430.

Buche S B, Dhondse S A, Khobragade A N, "Satellite image processing on parallel computing: A technical review”, Online International Conference on Green Engineering and Technologies, 2016, 1-9.

Codella N, Natsev A, Smith J R, "Towards large scale landcover recognition of satellite images”, 8th International Conference on Information, Communications and Signal Processing, 2011, pp1-5. 10.1109/ICICS.2011.6174291. Damahe L B, Thakur N V , "Review on Image Representation Compression and Retrieval Approaches", Technological Innovations in Knowledge Management and Decision Support 2019, DOI: 10.4018/978-1-52256164-4.ch009.

Distributed and Parallel computing, [online] Available: https://www.tutorialspoint.com/Distributed-Systems Haiming Z, Jianhui L, Yuanchun Z, Xuezhi W, Zhenghua X, Baoping Y, "Large-scale Storage and High-performance Processing Environment for Remote Sensor Images", Second International Conference on Networking and Distributed Computing, Beijing, 2011, pp 75-78, doi: 10.1109/ICNDC.2011.23.

High-Performance Computing(HPC),[online] Available: https://www.techopedia.com/definition/4595/high] Performance-computing-hpc.

Huiyu X, Hassan A K, Lingkui M, "Parallel implementation of Kaufman's initialization for clustering large remote sensing images on clouds", Computers, Environment and Urban Systems, Volume 61, Part B, 2017, pp 153-162, Landsat 5, [online] Available: Apache Spark, [online] Available: https://intellipaat.com/blog/tutorial/spark Tutorial.

Liu P “A Survey of Remote-sensing Big Data”, Frontiers in Environmental Science. 3. 2015, 10.3389/ fenvs.2015.00045.

Patel, H M, Panchal K, Chauhan P, Potdar M, "Large Scale Image Processing Using Distributed and Parallel Architecture- A survey",International Journal of Computer Science and Information Technologies, Vol. 6, 2015, pp 5531-5535.

Patel D D, Singh K. R, "Genome sequencing using MapReduce and Hadoop -A technical review. International Conference on Innovative Mechanisms for Industry Applications (ICIMIA), Bangalore, 2017, pp 544-547, doi: 10.1109/ICIMIA.2017.7975518.

Sharma T, Shokeen V, Mathur, S, “Distributed Processing of Satellite Images on Hadoop to Generate Normalized Difference Vegetation Index Images”, International Conference on Computing, Communication, Control and Automation (ICCUBEA), 2017, pp 1-5. 